\title{
Physicochemical Changes of the Gluten-Free Rice-Buckwheat Cookies during Storage - Artificial Neural Network Model
}

\author{
Mladenka Pestorić1, Marijana Sakač ${ }^{1}$, Lato Pezo², Dubravka Škrobot ${ }^{1}$, Nataša Nedeljković1, \\ Pavle Jovanov ${ }^{1}$, Bojana Filipčev ${ }^{1}$, Anamarija Mandić ${ }^{1}$ \\ Research Center for Technology of Plant Based Food Products, Institute of Food Technology, University of Novi Sad, \\ Bulevar Cara Lazara 1, 21000 Novi Sad, Serbia \\ 2 Engineering Department, Institute of General and Physical Chemistry, University of Belgrade, \\ Studentski trg 12-16, 11000 Belgrade, Serbia \\ *Corresponding author, e-mail: latopezo@yahoo.co.uk
}

Received: 18 September 2018, Accepted: 15 January 2019, Published online: 23 April 2019

\begin{abstract}
The influence of storage time, temperature, and packaging on some physicochemical characteristics of gluten-free rice-buckwheat cookies was studied. Shelf life markers, such as water activity $\left(a_{w}\right)$, hydroxymethylfurfural (HMF), firmness, and color parameters were modelled in relation to different storage conditions. Principal component analysis was applied to study the similarity among samples according to the observed parameters. The mathematical model in the form of an artificial neural network was developed to predict the physicochemical parameters of cookies during 6-month storage. The most evident differentiation among samples was observed for color coordinate $a^{*}, a_{w^{\prime}}$ and HMF. Regarding the methods for determination of the parameters, priority should be given to the instrumental determination of color as the most convenient method. The processing of experimental data allowed the creation of useful mathematical model to be used in predicting the behavior of physicochemical changes of cookies by different factor combinations during storage.
\end{abstract}

Keywords

gluten-free cookies, shelf life markers, mathematical model

\section{Introduction}

The best strategy for celiac disease patients is continuous adherence to gluten-free diet, which implies the strict utilization of gluten-free ingredients. Their incorporation in various food products has been studied by many authors [1]. In this regard, pseudocereals received a special attention [2].

The absence of structure forming gluten proteins in gluten-free formulation results in poor rheological and baking properties of gluten-free dough [3], and, therefore, production of high-quality gluten-free products represents a significant technological challenge, largely because of inadequate sensory properties of the final products [4]. Consequently, poor quality of gluten-free products can reflect on their shelf life, which has to be predicted.

Among gluten-free products, cookies represent one of the largest food categories, primarily due to their versatility, convenience, acceptable sensory properties, but especially because of their long shelf life. Even for a shelf-stable product, such as short dough cookies, there is a limitation on its shelf life due to deteriorating chemical reactions, determining its best-before date [5].

Physicochemical stability of cookies is primarily governed by their composition, which is characterized by relatively low water activity $\left(a_{w}\right)$ values and high fat content (20-30\% on the flour weight basis). The water activity of cookies is mainly lower than those that permit the growth of microorganisms $\left(a_{w}>0.6\right)$ [6], but this parameter is also important in terms of cookie sensory profiles. Although the moisture content does not exceed $7 \%$ in cookies, moisture migration affects cookie hardness, which represents a major problem in their shelf life.

Since cookies possess a high amount of vegetable fat, they are susceptible to oxidative changes, especially when they are exposed to conditions which favor lipid oxidation [7]. There are several approaches to predict cookie shelf life measuring lipid oxidation markers during storage 
- hexanal [8], heptanal [9], malonyldialdehyde (MDA) [10] or some other secondary lipid oxidation products [11].

During baking of cookies, Maillard reaction and caramelization also occur, resulting in the development of the desirable color and flavor of the final product. However, along with these substances, processing contaminants such as acrylamide and 5-hydroxymethylfurfural (HMF) are formed [12]. $H M F$, as a marker of non-enzymatic browning reaction, is directly related to the heat load applied during processing or storage [13]. It is known that $H M F$ was used for assessing the shelf life of a wide range of carbohydrate-containing foods such as cookies [12]. In addition, $H M F$ can also be presented in some cookie ingredients such as honey or caramel.

In addition, chemical deterioration reactions during storage can lead to increased levels of some degradation products of $H M F$ such as furan [14] or furan derivatives furfuryl aldehyde and 2-methylfurfural [13] meaning that mentioned substances can also serve as markers of food deterioration processes.

Our previous work [15] showed the possibility to predict the shelf life of the unpacked and packed gluten-free rice-buckwheat cookies, kept at ambient $\left(23 \pm 1{ }^{\circ} \mathrm{C}\right)$ and elevated $\left(40 \pm 1{ }^{\circ} \mathrm{C}\right)$ temperature during storage, measuring off-flavor volatile compounds (aldehydes), antioxidant capacity, total phenolic, rutin content, and evaluating sensory properties. Based on the obtained results, the evaluated sensory attributes were suggested to be the relevant parameters for predicting the endpoint of cookie shelf life. Despite the fact that sensory evaluation was assessed essential for cookie shelf life prediction [6], it is an expensive and longterm tool to conduct. Therefore, physicochemical parameters may play a crucial role in stability testing as they can be used either to predict the results obtained by the analytical sensory test or the endpoint of cookie shelf life.

Recently, mathematical modelling has been increasingly used for the study of shelf life. Developed empirical models show a reasonable fit to experimental data and successfully predict the shelf life of products [16]. Nonlinear models are found to be more suitable for real process simulation. Artificial neural network (ANN) models have gained momentum for modelling and control of processes [17].

ANN models are recognized as a good modelling tool since they provide the empirical solution to the problems from a set of experimental data, and are capable of handling complex systems with non-linearities and interactions between decision variables $[17,18]$.
The objective of this work was to study the physicochemical changes of gluten-free rice-buckwheat cookies as a function of temperature, storage time and packaging conditions. The investigation focused on the effectiveness of storage conditions on water activity, $H M F$, color, and firmness as the shelf life markers. Mathematical models, in the form of artificial neural network (ANN) were used for modelling and prediction of cookie storage process.

\section{Material and methods}

In our previous papers [15, 19], the used ingredients, preparation of cookies, as well as packaging and storage conditions of cookies were described in details. Therefore, in this paper they are shown in Fig. 1.

According to experimental plan, cookies were investigated monthly for changes in physicochemical parameters (during a six month period). Cookies were kept at (23 \pm $\left.1{ }^{\circ} \mathrm{C}\right)$ or $\left(40 \pm 1{ }^{\circ} \mathrm{C}\right)$, packed or unpacked, which makes $7 \times 2 \times 2=28$ samples used in this investigation.

\subsection{Water activity value}

Approximately $2.5 \mathrm{~g}$ of ground cookie sample were placed into the sample holder of an $a_{w}$-meter (TESTO 650, Testo AG, Lenzkirch, Germany) at $25^{\circ} \mathrm{C}$. Water activity $\left(a_{w}\right)$ values of three replicates were recorded after equilibration.

\subsection{Hydroxymethylfurfural $(H M F)$ analysis 2.2.1 Sample preparation}

The extraction procedure was performed according to Rufián-Henares et al. [20], with the modifications which were done by Petisca et al. [21]. Ten grams of sample were suspended in $5 \mathrm{~mL}$ water:methanol (70:30). The mixture was thoroughly stirred during $1 \mathrm{~min}$ and then $2.0 \mathrm{~mL}$ of Carrez I and Carrez II solutions were added and centrifuged at $5000 \mathrm{rpm}\left(4{ }^{\circ} \mathrm{C}\right)$ during $15 \mathrm{~min}$, recovering the supernatant to a $15 \mathrm{~mL}$ flask. Two more consecutive extractions were made with $2 \mathrm{~mL}$ of water:methanol (70:30) until collecting $10 \mathrm{~mL}$ of supernatant. Two milliliters of this solution were centrifuged at $8000 \mathrm{rpm}$ for 15 min before being analyzed.

\subsection{HPLC-DAD analysis}

The chromatographic separation and quantification of $H M F$ was performed using the HPLC method described by Ariffin et al. [22], and Tomasini et al. [23], with some modifications. The extracts were filtered through $0.45 \mu \mathrm{m}$ pore size nylon filter (Agilent Technologies, Santa Clara, CA, USA) before injection into the HPLC system. 


\section{PRODUCTION OF COOKIES}

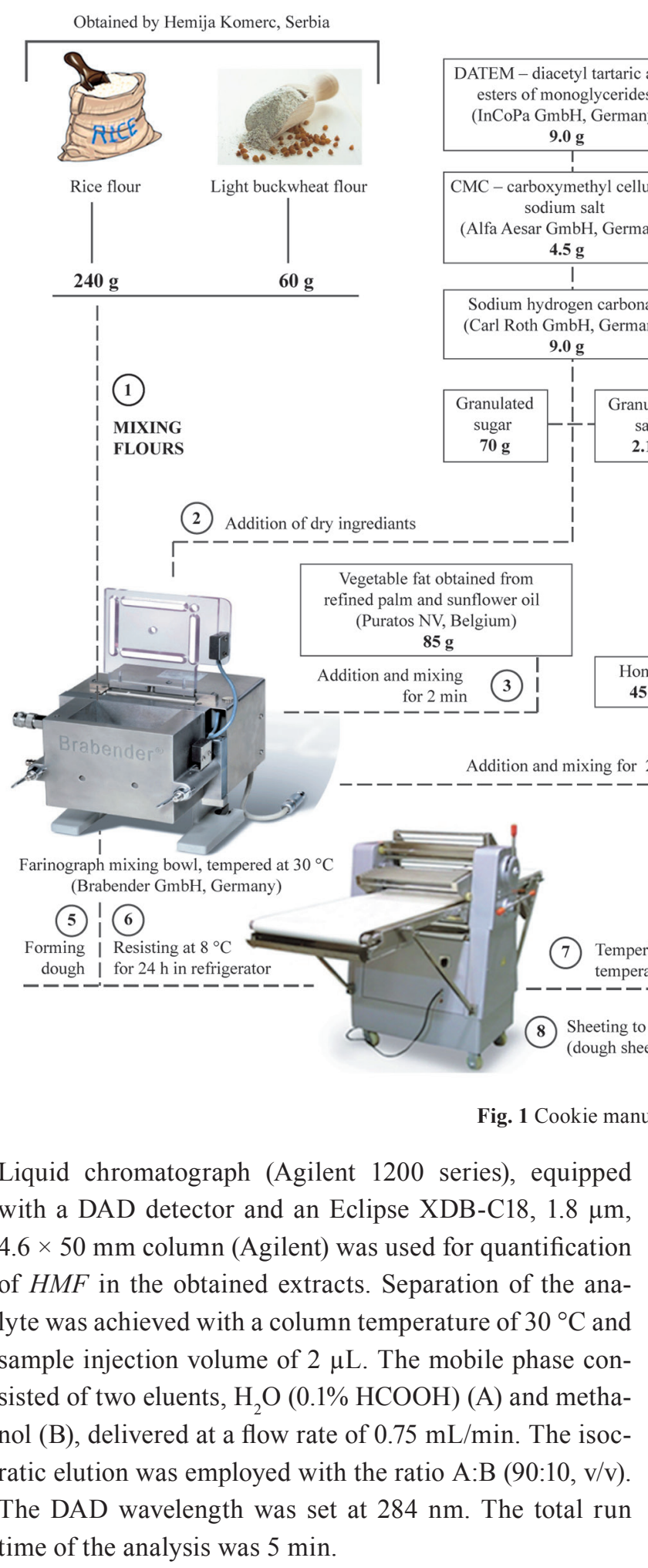

\subsection{Surface color determination}

Color was determined by a chromameter Minolta, type CR 400 (Minolta Co., Ltd., Osaka, Japan) on the top surface

\section{PACKAGING AND STORAGE}

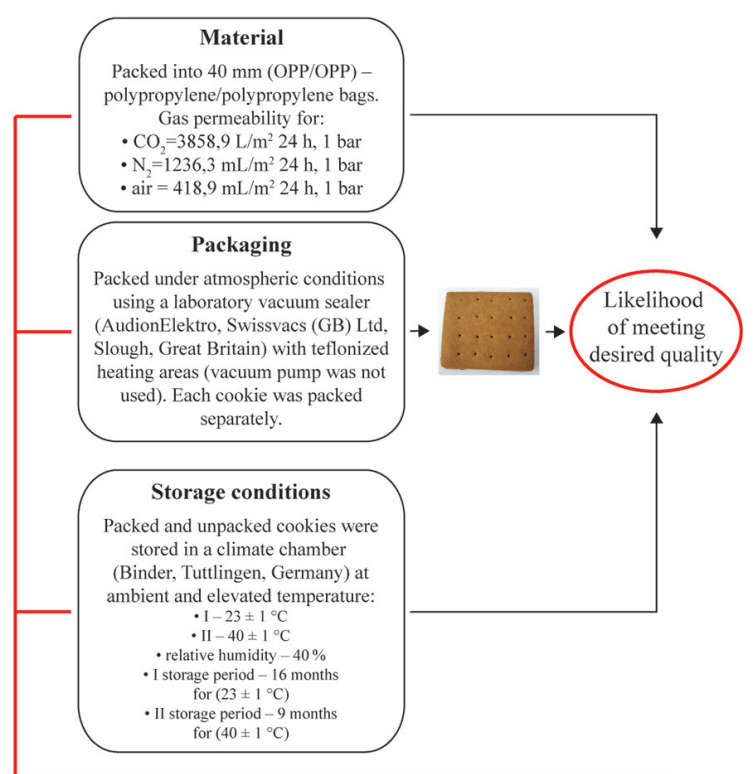

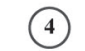

(4) Deionized water $5 \mathrm{~g}$ I

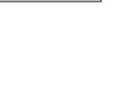


According to the results of PCA analysis, $\Delta E^{*}$ could be used only for prediction of shelf life, while the $L^{*} a^{*} b^{*}$ color system could be used for more detailed shelf life prediction which include the prediction of possibility of microbial spoilage and the increase of $H M F$ amount. Both $\Delta E^{*}$ and $B I$ show the changes in color of cookies during the shelf life period (which are also described by the dominant color changes in $L^{*}$ and $b^{*}$ color coordinates), while the changes in storage conditions (packaging and temperature) influence $a^{*}$ color coordinate. The subscript ' 0 ' indicates the initial color of the cookie sample.

\subsection{Textural measurement}

Textural analysis was conducted using a TA.XTPlus Texture Analyzer (Stable Micro Systems Ltd., Surrey, UK), equipped with a 3-point bending rig ( $\mathrm{HDP} / 3 \mathrm{~PB})$, and a $5 \mathrm{~kg}$ load cell. Texture analyzer settings were: mode-measure force in compression; pre-test speed $1.0 \mathrm{~mm} / \mathrm{s}$; test speed $3.0 \mathrm{~mm} / \mathrm{s}$; post-test speed $10.0 \mathrm{~mm} / \mathrm{s}$; distance $5.0 \mathrm{~mm}$; trigger force $50 \mathrm{~g}$. Cookie hardness/firmness expressed as a peak force $(F)$ at the time of interruption (the point of break) was determined. Ten measurements per each sample were performed.

\subsection{Statistical analysis}

The analysis of variance (ANOVA) was applied for the comparison of means of the collected data, and significant differences were determined according to post-hoc Tukey's HSD test at $p<0.05$ significance level. Principal component analysis (PCA) was enforced to the experimental data to characterize the internal relations between the variables and to differentiate the investigated samples. All data were processed statistically using the software package STATISTICA 10.0 (StatSoft Inc., Tulsa, OK, USA).

\subsubsection{Artificial neural network (ANN) modelling}

The ANN model in the form of multilayer perceptron model (MLP), (with input, hidden and output layers) was developed for prediction of the changes in gluten-free rice-buckwheat cookies during storage. This form of model has been proven in approximating nonlinear functions [25]. Input and output data were normalized prior to ANN calculation in order to improve the behavior of the ANN. BroydenFletcher-Goldfarb-Shanno (BFGS) algorithm was used for network optimization [26]. The experimental results used for ANN modelling were randomly divided into: training (60\%), cross-validation ( $20 \%$ ) and testing data (20\%). The training data set was used for the learning cycle of ANN, assuming that the successful training was achieved when learning and cross-validation curves approached zero. The coefficients associated with the hidden layer and the output of the ANN model was determined during the training cycle, in which the BFGS algorithm was used as an optimization procedure to minimize the error between network and experimental outputs [26].

\subsubsection{Sensitivity analysis}

Sensitivity analysis was used to investigate the influence of input variables on the observed outputs, evaluated at specific centile points for each input variable [27]. This analysis is also necessary to check if the ANN could behave erroneously [28]. The infinitesimal amount has been added to each input variable in 10 equally spaced individual points determined by the minimum and maximum of the training data to check the influence of input variables on the observed outputs [27].

\subsubsection{The accuracy of the models}

The adequacy of the developed models was tested using coefficient of determination $\left(r^{2}\right)$, reduced chi-square $\left(\chi^{2}\right)$, mean bias error $(M B E)$, root mean square error (RMSE) and mean percentage error (MPE).

\section{Results and discussion}

The unpacked and packed gluten-free rice-buckwheat cookies were studied during storage at ambient $\left(23 \pm 1^{\circ} \mathrm{C}\right)$ and elevated $\left(40 \pm 1{ }^{\circ} \mathrm{C}\right)$ temperature, in terms of chemical parameters $\left(a_{w}\right.$ and $\left.H M F\right)$, color values $\left(L^{*}, a^{*}, b^{*}, h^{*}, C^{*}\right.$, $\left.\Delta E^{*}\right)$, and texture firmness $(F)$.

The impact of ingredients, manufacturing process, packaging, and storage conditions on the cookie quality during the time was described in the previous paper [15].

PCA was applied to characterize and differentiate between the investigated samples, according to observed parameters. The PCA of the presented data explained that the first two principal components accounted for $70.73 \%$ of the total variance $(52.74 \%$ and $17.99 \%$, respectively), in the nine variables factor space $\left(a_{w}, H M F, L^{*}, a^{*}, b^{*}\right.$, $C^{*}, h^{*}, \Delta \mathrm{E}^{*}$ and $\left.F\right)$. Considering the map of the PCA performed on the data, the observed variables, with the contribution of the total variance of $17.3 \%$ for $L^{*}, 16.4 \%$ for $b^{*}, 15.9 \%$ for $\Delta E^{*}, 14.1 \%$ for $C^{*}$, and $19.95 \%$ for $h^{*}$, exhibited negative scores according to first principal component (PC1) (Fig. 2). The negative contribution to the second principal component calculation was observed for $a_{w}$ with a $20.7 \%$ contribution to the total variance, $a^{*}$ with 


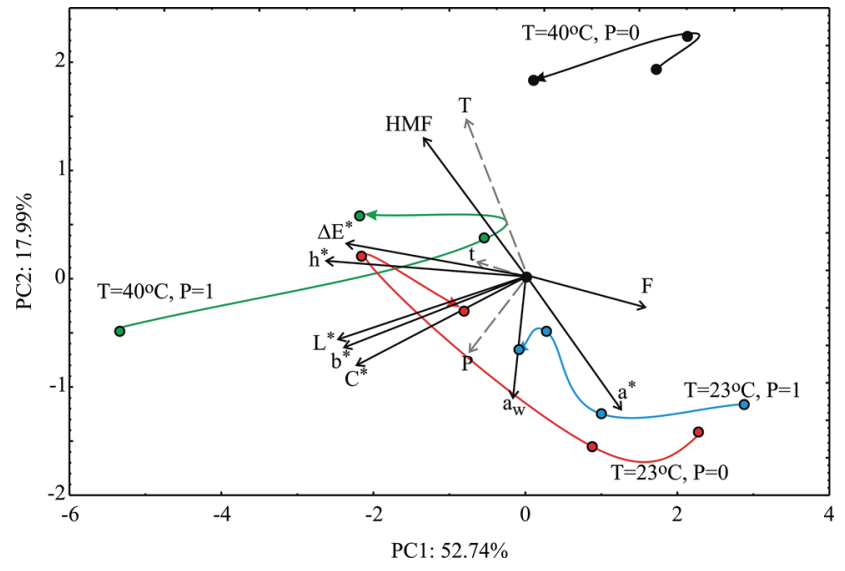

Fig. 2 Principal component analysis (PCA) ordination of all observed variables for gluten-free rice-buckwheat cookies based on component correlations (unpacked $(P=0)$ and packed $(P=1)$ gluten-free ricebuckwheat cookies during 6-month storage $(t)$ at two different temperatures $(T)\left(23 \pm 1{ }^{\circ} \mathrm{C}\right.$ and $\left.40 \pm 1{ }^{\circ} \mathrm{C}\right)$

a $24.9 \%$ contribution to the total variance, and $C^{*}$ with a $10.5 \%$ contribution to the total variance. The most positive influence was observed for $H M F$ with a $28.8 \%$ contribution to the total variance.

According to the orientation of the vector describing $H M F$ in the factor space, the content of $H M F$ is more pronounced for samples kept at a higher temperature (upper part of the PCA graphic). These samples had lower values of $a^{*}$ color coordinate (more greenish samples) and lower $a_{w}$ values, indicating the reduced potential for microbiological spoilage. HMF and $a^{*}$ color coordinate are oppositionally oriented on the graph, explaining the negative correlation between these variables. The similar direction of $L^{*}$ and $b^{*}$ color coordinates indicates that packed samples kept at $40{ }^{\circ} \mathrm{C}$ at the beginning of the storage period were more yellowish and lighter than other samples. The angle between $L^{*}$ and $b^{*}$ color coordinate vectors indicates the high degree of correlation, as well as the high correlation to color coordinates $h^{*}, C^{*}$, and $\Delta E^{*}$. Color coordinates $L^{*}$ and $b^{*}$ were negatively correlated to firmness $(F)$. According to the obtained results, the observed samples became less acceptable during the storage, regardless of packaging condition.

\subsection{Artificial neural network model}

The optimization procedures for minimizing the error function between network and experimental outputs were used during the ANN learning cycle [26, 28]. The optimum number of hidden neurons was chosen upon minimizing the difference between predicted ANN values and experimental results. According to ANN performance, it was noticed that the optimal number of neurons in the hidden layer for $a_{w}, F, L^{*}, a^{*}, b^{*}, \Delta E^{*}, C^{*}, h^{*}$, and $H M F$ calculation was 6 (network MLP 3-6-9) to obtain high values of $r^{2}$ (overall 0.898 for ANN during the considering period), and low values of the sum of squares (SOS) (Table 1).

Furthermore, ANN models were used to predict experimental variables $\left(a_{w}, F, L^{*}, a^{*}, b^{*}, \Delta E^{*}, C^{*}, h^{*}\right.$, and $\left.H M F\right)$ (Fig. 3).

The predicted values were very close to the desired values in most cases in terms of $r^{2}$ value of ANN models. SOS obtained with ANN models are of the same order of magnitude as experimental errors for $a_{w}, F, L^{*}, a^{*}, b^{*}, \Delta E^{*}$, $C^{*}, h^{*}$, and $H M F$, reported in the literature earlier [26, 29].

\subsection{Sensitivity analysis}

The influence of the input over the output variables, i.e. calculated changes of the output variables for infinitesimal changes in the input variables are shown in Fig. 4.

As it can be seen in Fig. $4, L^{*}, b^{*}, \Delta E^{*}, C^{*}$ and $h^{*}$ color coordinates were most affected by the infinitesimal changes in temperature at the minimum values of the input space. The content of $H M F$ was most affected by temperature changes at the maximum values of the input space, i.e. the contents of $H M F$ increased throughout the storage time at the higher temperatures. $a_{w}$ and $F$ were most affected by temperature changes at the lower end of the input space. The influence of storage time was more expressed at the center of the input space for the calculation of $a_{w}, F, L^{*}, b^{*}, \Delta E^{*}, C^{*}$ color coordinates, and $H M F$ content, while the influence of infinitesimal changes in the storage time was more observable for lower values in the input space for $a^{*}$ and $h^{*}$ color coordinates.

\begin{tabular}{lc} 
Table 1 Artificial neural network ANN performance summary \\
\hline Network name & MLP 3-6-9 \\
\hline Training's performance & 0.899 \\
Test performance & 0.778 \\
Validation performance & 0.556 \\
Training error & 0.075 \\
Test error & 0.261 \\
Validation error & 1.146 \\
Training algorithm & BFGS 24 \\
Error function & SOS \\
Hidden activation & Tanh \\
Output activation & Exponential \\
\hline *Tanh - Hyperbolic tangent, SOS - sum of squares
\end{tabular}



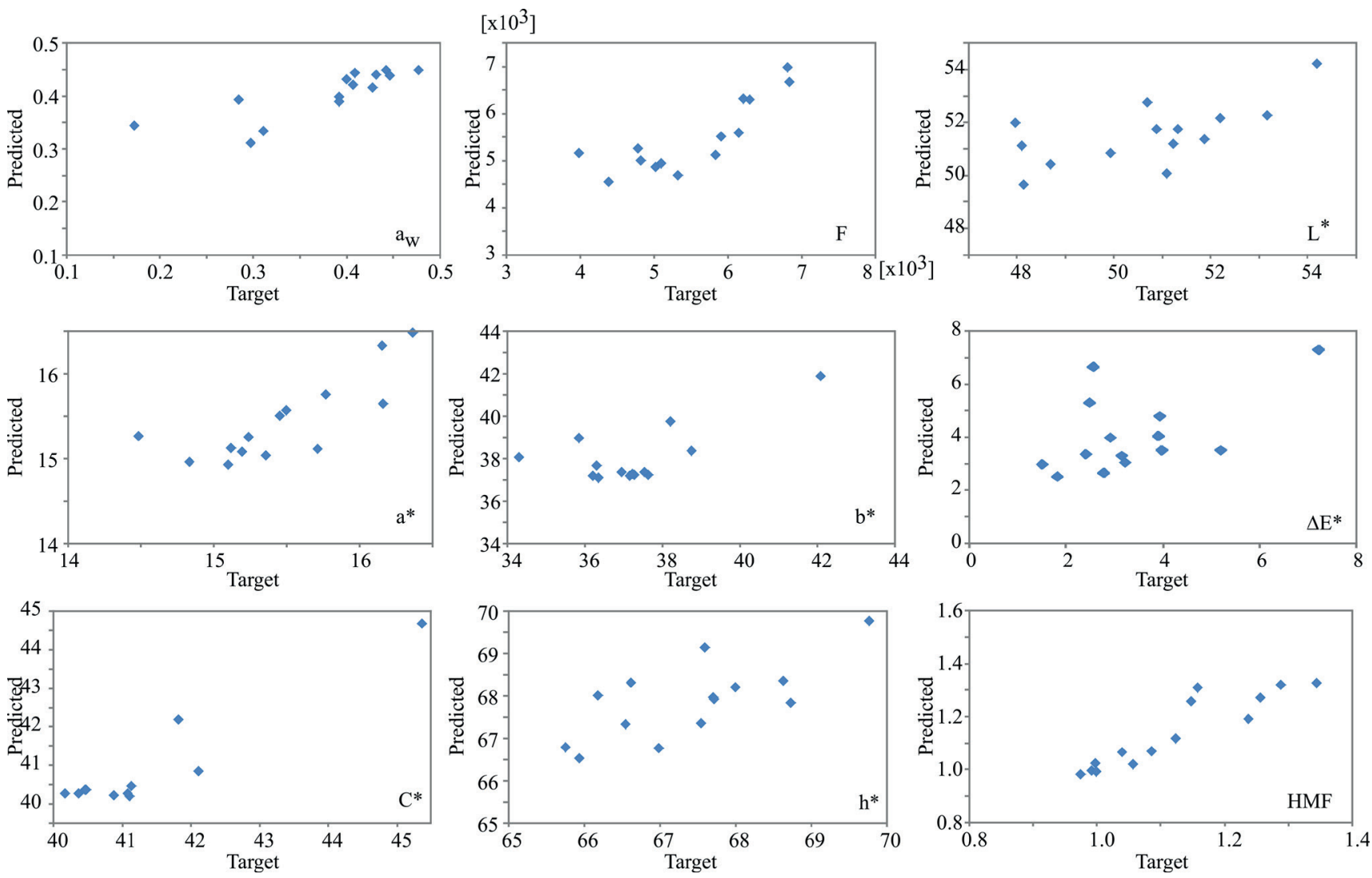

Fig. 3 Experimentally measured and the ANN model predicted values of all observed variables

Table 2 The 'goodness of fit' tests of the developed mathematical models

\begin{tabular}{lcccccccccc}
\hline & $\chi^{2}$ & $R M S E$ & $M B E$ & $M P E$ & $r^{2}$ & Skewww. & Kurt & Mean & SD & Var. \\
\hline$a_{w}$ & 0.05 & 0.12 & $6.04 \cdot 10^{-16}$ & 70.64 & $0.81^{*}$ & 0.30 & 3.33 & $8.62 \cdot 10^{-17}$ & 0.04 \\
$H M F$ & 0.13 & 0.18 & $1.11 \cdot 10^{-16}$ & 34.44 & $0.84^{+}$ & -0.67 & 1.14 & $1.59 \cdot 10^{-17}$ & 0.07 & 0.00 \\
$L^{*}$ & 22.76 & 2.39 & $2.84 \cdot 10^{-14}$ & 10.86 & $0.95^{+}$ & 0.23 & 0.21 & $3.79 \cdot 10^{-15}$ & 0.89 \\
$a^{*}$ & 1.67 & 0.65 & $-2.00 \cdot 10^{-14}$ & 8.02 & $0.97^{+}$ & -1.31 & 3.65 & $-2.66 \cdot 10^{-15}$ & 0.24 & 0.78 \\
$b^{*}$ & 15.61 & 1.98 & $9.59 \cdot 10^{-14}$ & 11.88 & $0.91^{+}$ & 0.00 & -0.03 & $1.28 \cdot 10^{-14}$ & 0.73 \\
$C^{*}$ & 13.05 & 1.81 & $-7.11 \cdot 10^{-14}$ & 9.92 & $0.86^{+}$ & 0.10 & -0.24 & $-9.47 \cdot 10^{-15}$ & 0.67 \\
$h^{*}$ & 8.13 & 1.43 & $-8.17 \cdot 10^{-14}$ & 4.70 & $0.97^{+}$ & 0.12 & -0.11 & $-1.09 \cdot 10^{-14}$ & 0.53 \\
$\Delta E$ & $4.2 \cdot 10^{1}$ & 3.3 & $2.1 \cdot 10^{-14}$ & $4.3 \cdot 10^{3}$ & $0.76^{*}$ & 0.350 & 2.322 & 0.000 & 0.45 \\
$F$ & $2.85 \cdot 10^{6}$ & 843.50 & $-4.2 \cdot 10^{-12}$ & 26.74 & $0.83^{*}$ & -0.57 & 3.25 & $-8.01 \cdot 10^{-13}$ & 377.23 & $1.42 \cdot 10^{5}$ \\
\hline
\end{tabular}

$\chi^{2}$ - reduced chi-square; $R M S E$ - root mean square error; $M B E$ - mean bias error; $M P E$ - mean percentage error; Skew. - skewness; Kurt. - Kurtosis; SD - standard deviation; Var. - variance. ${ }^{+}$model is statistically significant at $p<0.01$ level; ${ }^{*}$ model is statistically significant at $p<0.05$ level.

\section{3 'Goodness of fit' tests and residual analysis}

The common 'goodness of fit' tests and residual analysis were performed [27] and the obtained results are shown in Table 2.

The obtained $r^{2}$ values were high, and $\chi^{2}, M B E, R M S E$, and $M P E$ were low, indicating that this model generally shows a good fit to experimental data in the experimental domain at which points were not included in the regression, as mentioned earlier by Madamba [30].

\section{Conclusions}

Assessing the characterization and differentiation patterns based on PCA analysis, it can be concluded that cookies became significantly brighter during storage, i.e. longer storage caused significant changes in the cookie color. At the same time, storage at ambient temperature led to an increase in $a_{w}$ value, and as time passed, the samples became softer, in contrast to the samples kept at a higher temperature. The content of $H M F$ was more pronounced 
[x106]

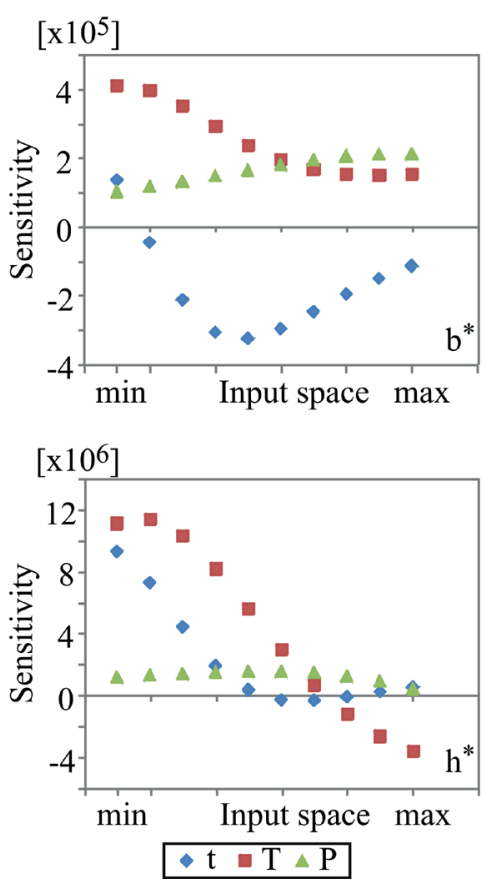
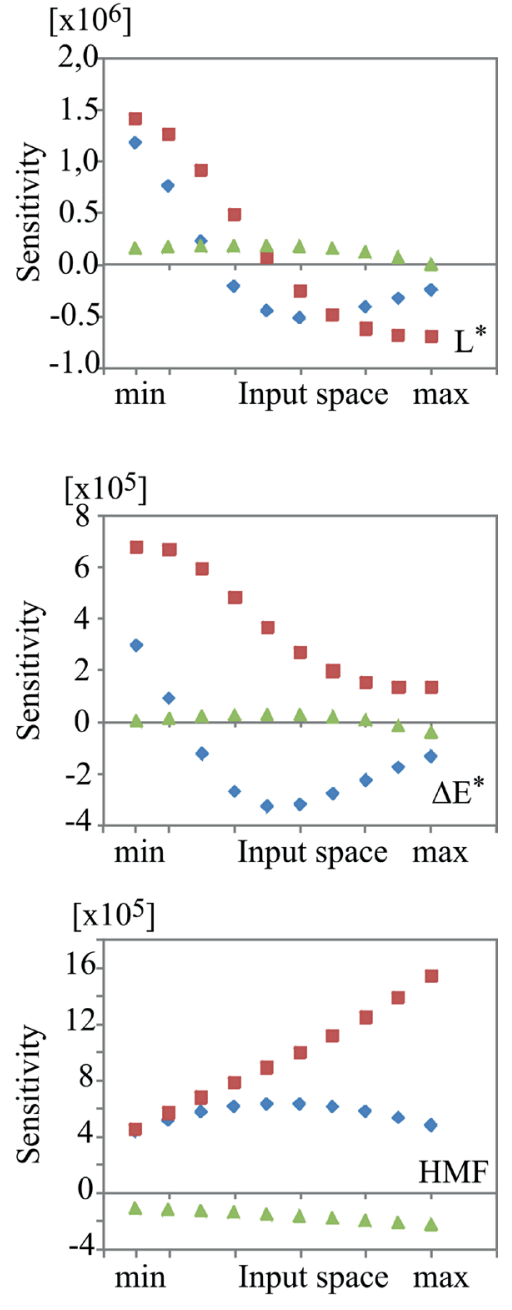

Fig. 4 Sensitivity analysis - the influence of the input over the output variables $T$-storage temperature; $t$ - storage time; $P$ - logical content regarding the packing state of cookies (packed or unpacked)

for samples kept at a higher temperature. These samples were also characterized by lower $a^{*}$ and $a_{w}$ value. $L^{*}$ and $b^{*}$ color coordinates were highly correlated for all samples and these variables were negatively correlated to firmness. According to the obtained results, samples became less acceptable during the storage, regardless of the packaging condition. Depending on the storage conditions, the best differentiation among samples was achieved on the basis of parameters $a^{*}, a_{w}$, and $H M F$. Regarding the methods for determination of these parameters, priority should be given to the instrumental determination of color as the easiest way to monitor the changes during the cookie storage. The results of this study showed that the processing of experimental data allowed the creation of useful mathematical model to be used in predicting the behavior of physicochemical changes of cookie samples by different factor combinations during storage. The predicted values fitted well to the experimental ones in most cases, according to the 'goodness of fit' tests for the developed mathematical model, and can be successfully used for prediction in controlling the storage process of this type of product.

\section{Acknowledgements}

This work was financially supported by the Ministry of Education, Science and Technological Development, Republic of Serbia (Project No. TR31029). 


\section{References}

[1] Hoube, A., Höchstötter, A., Becker, T. "Possibilities to increase the quality in gluten-free bread production: an overview", European Food Research and Technology, 235(2), pp. 195-208, 2012. https://doi.org/10.1007/s00217-012-1720-0

[2] Alvarez-Jubete, L., Arendt, E.K., Gallagher, E. "Nutritive value of pseudocereals and their increasing use as functional gluten-free ingredients", Trends in Food Science and Technology, 21(2), pp. 106-113, 2010. https://doi.org/10.1016/j.tifs.2009.10.014

[3] Pruska-Kedzior, A., Kedzior, Z., Goracy, M., Pietrowska, K., Przybylska, A., Spychalska, K. "Comparison of rheological, fermentative and baking properties of gluten-free dough formulations", European Food Research and Technology, 227, pp. 15231536, 2008. https://doi.org/10.1007/s00217-008-0875-1

[4] da Silva, T. F., Conti-Silva, A. C. "Preference mappings for gluten-free chocolate cookies: Sensory and physical characteristics", Nutrition and Food Science, 46(3), pp. 374-387, 2016. https://oi.org/10.1108/NFS-11-2015-0139

[5] Wibowo, S., Grauwet, T., Gedefa, G. B., Hendrickx M., Van Loey, A. "Quality changes of pasteurised mango juice during storage. Part I: Selecting shelf-life markers by integration of a targeted and untargeted multivariate approach", Food Research International, 78, pp. 396-409, 2015.

https://doi.org/10.1016/j.foodres.2015.09.002

[6] Chieh, C. "Water", In: Hui, Y.H. (ed.) Bakery Products Science and Technology, Blackwell Publishing, Oxford, UK, pp. 211232, 2006. [online] Available at: https://archive.org/stream/ BakeryProductsScienceAndTechnologyWeibiaoZ/Bakery_ Products_Science_and_Technology_-_Weibiao_Z_djvu.txt [Accessed: 10 January 2018]

[7] Zieliński, H., del Castillo, M. D., Prygodzka, M., Ciesarova, Z., Kukurova, K., Zielińska, D. "Changes in chemical composition and antioxidative properties of rye ginger cakes during their shelflife", Food Chemistry, 135(4), pp. 2965-2973, 2012. https://doi.org/10.1016/j.foodchem.2012.07.009

[8] Berenzon, S., Saguy, I. S. "Oxygen absorbers for extension of crackers shelf-life", LWT-Food Science and Technology, 31(1), pp. 1-5, 1998.

https://doi.org/10.1006/fstl.1997.0286

[9] Viscidi, K. A., Dougherty, M. P., Briggs, J. L., Camire, M. E. "Complex phenolic compounds reduce lipid oxidation in extruded oat cereals", LWT-Food Science and Technology, 37(7), pp. 789796, 2004.

https://doi.org/10.1016/j.lwt.2004.03.005

[10] Sakač, M., Pezo, L., Jovanov, P., Nedeljković, N., Mandić, A., Pestorić, M., Mišan, A. "Application of the SHS-GC-FID method and HPLC-DAD method in the prediction of the shelf life of gluten-free cookies", Journal of the Serbian Chemical Society, 82(12), pp. 1343-1355, 2017.

https://doi.org/10.2298/JSC170421074S
[11] Yang, N., Hort, J., Linforth, R., Brown, K., Walsh, S., Fisk, I. D. "Impact of flavour solvent (propylene glycol or triacetin) on vanillin, 5-(hydroxymethyl)furfural, 2,4-decadienal, 2,4-heptadienal, structural parameters and sensory perception of shortcake biscuits over accelerated shelf life testing", Food Chemistry, 141(2), pp. 1354-1360, 2013. https://doi.org/10.1016/j.foodchem.2013.03.084

[12] Van Der Fels-Klerx, H. J., Capuanoa, E., Nguyena, H. T., Ataç Mogolb, B., Kocadağlı, T., Göncüoğlu Taş, N., Hamzalığlu, A., Van Boekel, M. A. J. S., Gökmen, V. "Acrylamide and 5-hydroxymethylfurfural formation during baking of biscuits: $\mathrm{NaCl}$ and temperature - time profile effects and kinetics", Food Research International, 57, pp. 210-217, 2014. https://doi.org/10.1016/j.foodres.2014.01.039

[13] Ameur, A. L., Trystram, G., Birlouez-Aragon, I. "Accumulation of 5-hydroxymethyl-2-furfural in cookies during the backing process: Validation of an extraction method", Food Chemistry, 98(4), pp. 790-796, 2006. https://doi.org/10.1016/j.foodchem.2005.07.038

[14] Palmers, S., Grauwet, T., Buvé, C., Van de Vondel, L., Kebede, B. T., Hendrickx, M. E., Van Loey, A. "Furan formation during storage and reheating of sterilised vegetable purées", Food Additives and Contaminants: Part A, 32(2), pp. 161-169, 2015. https://doi.org/10.1080/19440049.2014.999720

[15] Sakač, M., Pestorić, M., Mandić, A., Mišan, A., Nedeljković, N., Jambrec, D., Jovanov, P., Lazić, V., Pezo, L., Sedej, I. "Shelf-life prediction of gluten-free rice-buckwheat cookies", Journal of Cereal Science, 69, pp. 336-343, 2016. https://doi.org/10.1016/j.jcs.2016.04.008

[16] Siripatrawan, U., Makino, Y. "Simultaneous assessment of various quality attributes and shelf life of packaged bratwurst using hyperspectral imaging", Meat Science, 146, pp. 26-33, 2018. https://doi.org/10.1016/j.meatsci.2018.06.024

[17] Zhang, Y. "Application of improved BP neural network based on e-commerce supply chain network data in the forecast of aquatic product export volume", Cognitive Systems Research, In press, 2018.

https://doi.org/10.1016/j.cogsys.2018.10.025

[18] Siripatrawan, U., Jantawat, P. "A novel method for shelf life prediction of a packaged moisture sensitive snack using multilayer perceptron neural network", Expert Systems with Applications, 34(2), pp. 1562-1567, 2008.

https://doi.org/10.1016/j.eswa.2007.01.008

[19] Sakač, M., Pestorić, M., Misan, A., Nedeljković, N., Jambrec, D., Jovanov, P., Banjac, V., Torbica, A., Hadnđev, M., Mandić, A. "Antioxidant capacity, mineral content and sensory properties of gluten-free rice and buckwheat cookies", Food Technology and Biotechnology, 53(1), pp. 38-47, 2015. https://doi.org/10.17113/ftb.53.01.15.3633 
[20] Rufián-Henares, J. A., Andrade, C. D., Morales, F. J. "Application of a fast high-performance liquid chromatography method for simultaneous determination of furanic compounds and glucosylisomaltol in breakfast cereals", Journal of AOAC International, 89(1), pp. 161165, 2006. [online] Available at: https://www.ingentaconnect.com/content/aoac/jaoac/2006/00000089/00000001/art00022 [Accessed: 10 January 2018]

[21] Petisca, C., Henriques, A. R., Pérez-Palacios, T., Pinho, O., Ferreira, I .M. P. L. V. O. "Assessment of hydroxymethylfurfural and furfural in commercial bakery products", Journal of Food Composition and Analysis, 33(1), pp. 20-25, 2014. https://doi.org/10.1016/j.jfca.2013.10.004

[22] Ariffin, A. A., Ghazali, H. M., Kavousi, P. "Validation of a HPLC method for determination of hydroxymethylfurfural in crude palm oil", Food Chemistry, 154, pp. 102-107, 2014.

https://doi.org/10.1016/j.foodchem.2013.12.082

[23] Tomasini, D., Sampaio, M. R. F., Caldas, S. S., Buffon, J. G., Duarte, F. A., Primel, E. G. "Simultaneous determination of pesticides and 5-hydroxymethylfurfural in honey by the modified QuEChERS method and liquid chromatography coupled to tandem mass spectrometry", Talanta, 99, pp. 380-386, 2012. https://doi.org/10.1016/j.talanta.2012.05.068

[24] Buera, M. P., Retriella, C., Lozano, R. D. "Definition of color in the nonenzymatic browning", Die Farbe, 33, pp. 316-326, 1985.

[25] Karlović, S., Bosiljkov, T., Brnčić, M., Ježek, D., Tripalo, B., Dujmić, F., Džineva, I., Skupnjak, A. "Comparison of artificial neural network and mathematical models for drying of apple slices pretreated with high intensity ultrasound", Bulgarian Journal of Agricultural Sciences, 19(6), pp. 1372-1377, 2013. [online] Available at: https://www.agrojournal.org/19/06-30.pdf [Accessed: 10 January 2018]
[26] Pezo, L. L., Ćurčić, B. L., Filipović, V. S., Nićetin, M. R., Koprivica, G. B., Mišljenović, N. M., Lević, L. B. "Artificial neural network model of pork meat cubes osmotic dehydratation", Hemijska industrija, 67(3), pp. 465-475, 2013. https://doi.org/10.2298/HEMIND120529082P

[27] Pezo, L. L., Čolović, R. R., Palić, D. V., Jovanović, A. P. "Use of different statistical approaches in prediction of metabolizable energy of diets for broilers", Food and Feed Research, 42, pp. 73-81, 2015. https://doi.org/10.5937/FFR1501073P

[28] Taylor, B. J. "Methods and Procedures for the Verification and Validation of Artificial Neural Networks", 1st ed., Springer Science and Business Media, Inc., New York, USA, 2006. https://doi.org/10.1007/0-387-29485-6

[29] Chattopadhyaya, P. B., Rangarajana, R. "Application of ANN in sketching spatial nonlinearity of unconfined aquifer in agricultural basin", Agricultural Water Management, 133, pp. 81-91, 2014. https://doi.org/10.1016/j.agwat.2013.11.007

[30] Madamba, P. S. "The response surface methodology: an application to optimize dehydration operations of selected agricultural crops", LWT - Food Science and Technology, 35(7), pp. 584-592, 2002.

https://doi.org/10.1006/fstl.2002.0914 